\title{
Familial adenomatous polyposis with early onset dysplasia in a Bangladeshi girl: a case report
}

\author{
KHAN LAMIA NAHID ${ }^{1}$, MD. RUKUNUZZAMAN² ${ }^{2}$ MUKESH KHADGA ${ }^{3}$, FAHMIDA BEGUM ${ }^{1}$, \\ ASM BAZLUL KARIM ${ }^{2}$
}

\begin{abstract}
:
Familial adenomatous polyposis (FAP) is characterized by hundreds to thousands of adenomatous polyps in the colon which usually starts at adolescence. Familial adenomatous polyposis (FAP) is a rare, autosomal dominant condition caused by a defect in the adenomatous polyposis coli (APC) gene. FAP arises from germline mutations of the APC gene on chromosome $5 q 21$. If left untreated, all patients will develop colon cancer by age 35-40 years. Their lifetime risk of developing colorectal cancer is estimated to exceed $99 \%$ in patients who do not undergo a colectomy. Eventually, there is increased risk of development of other malignancies. Early surgical removal of colon can greatly reduce the spread of cancer. We reported a case of FAP whose symptoms started at early age. Early dysplastic changes were found on histological report. She had undergone prophylactic colectomy. Though appropriate timing for colectomy is late teens, she had surgery much earlier time. Ileal pouch anal anastomosis was done instead of lleorectal anastomosis. Large sized polyps were the indication of preponed colectomy. But lifelong surveillance is necessary for the girl to detect other malignancies after colectomy.
\end{abstract}

Key Words: Familial adenomatous polyposis, adenomatous polyposis coli, ileorectal anastomosis, ileal pouch anal anastomosis.

Introduction:

Familial adenomatous polyposis (FAP) is a rare, autosomal dominant inherited condition caused by a defect in the adenomatous polyposis coli (APC) gene. FAP arises from germline mutations of the $A P C$ gene on chromosome $5 q 21 .{ }^{1}$ The APC is a tumour suppressor gene responsible for the production of adenomatous polyposis coli (APC), a large multifunction tumor-suppressing protein which acts as a "gatekeeper" to prevent development of tumors. APC regulates â-catenin, a protein that plays a crucial role in cell communication, signaling, growth, and controlled destruction, but which left uncontrolled also gives rise to numerous cancers. ${ }^{2,3}$ Although the polyps are inherently benign, the first step of the two-

1. Assistant Professor, Dept. of Paediatric Gastroenterology and Nutrition, Bangabandhu Sheikh Mujib Medical Univrsity (BSMMU), Dhaka.

2. Professor, Dept. of Paediatric Gastroenterology and Nutrition, BSMMU, Dhaka.

3. Resident Phase B, Dept. of Paediatric Gastroenterology and Nutrition, BSMMU, Dhaka.

Corresponding Author: Dr Khan Lamia Nahid, Assistant Professor, Department of Paediatric Gastroenterology \& Nutrition, BSMMU, Dhaka. Cell: 01711362681, email: lamianahid@ yahoo.com

Received: 06/04/2019 Acceepted: 20/05/2019 hit hypothesis has already taken place: the inherited APC mutation. Often, the remaining "normal" allele is mutated or deleted, accelerating generation of polyps. Further mutations (e.g., in p53 or kRAS) to APCmutated cells are much more likely to lead to cancer than they would in non-mutated epithelial cell. The genetic mutation may also occur spontaneously for 25 to 30 percent of people. FAP affects males and females in equal numbers. Estimates of the prevalence of FAP vary from 1 in 6,850 to 1 in 31,250 live births ( 2.29 to 3.2 cases per 100,000 individuals) ${ }^{4}$ It occurs in approximately one in 5,000 to 10,000 individuals in the United States and accounts for about $0.5 \%$ of all cases of colorectal cancer. The condition is characterized by the early onset of numerous adenomatous polyps throughout the colon. If left untreated, all patients will develop colon cancer by age $35-40$ years. Their lifetime risk of developing CRC (colorectal cancer) is estimated to exceed $99 \%$ in patients who do not undergo a colectomy. ${ }^{5}$ Eventually, there is increased risk of development of other malignancies. Early surgical removal of colon can greatly reduce the spread of cancer. $^{6}$ 


\section{Case report:}

A twelve year old girl of nonconsanguineous parents got admitted in Bangabandhu Sheikh Mujib Medical University (BSMMU) on October, 2018 with the complaints of painless intermittent per rectal bleeding for six years. The bleeding was fresh, small in amount, occurring drop by drop, usually after defecation. She had no history of abdominal pain, fever, altered bowel habit, vomiting or something coming down per rectum. On query, mother gave history of colonoscopy two years back. She was also transfused with total five units of fresh blood. Her mother also had similar type of illness and mother's colonoscopic report showed adenomatous polyps. On physical examination, the girl was well alert, mildly pale, normothermic, normotensive (BP- 100/60 mm of $\mathrm{Hg}$ ). Skin survey revealed normal findings. Oral cavity was normal. Per abdominal examination showed no organomegaly. On digital rectal examination, a polypoid mass was felt at finger tip on posterior rectal wall approximately $5 \mathrm{~cm}$ from anal verge. Laboratory investigations showed that complete blood count was normal. On colonoscopy, polyps of variable sizes (from $1 \mathrm{~cm}$ to $15 \mathrm{~cm}$ ) were seen over entire rectosigmoid region and also descending colon. Transverse colon, ascending colon and caecum were normal. Polypectomy of three large polyps were done including one large fungiform polyp. Multiple biopsies were taken from polyps. Tubulovillous adenoma with low grade dysplasia was found in her histopathology report. Patient was investigated for FAP assosciated lesions. Fundoscopy was done to exclude CHRPE (congenital hypertrophy of retinal pigment epithelium). Upper gastrointestnal endoscopy was done to exclude fundic gastric polyps and other tumors. Both examinations were found normal. As genetic test is not possible in our country for APC gene, so diagnosis is not confirmed for Familial adenomatous polyposis. But in this case, diagnosis is made by family history, typical clinical features and histological findings. She was referred to surgery department for prophylactic colectomy. It was done to prevent further development of colon cancer. Three months later, procto colectomy with ileal pouch anal anastomosis (IPAA) instead of ileorectal anastomosis (IRA) was performed. Histopathology of excised colonic polyps again revealed tubular adenoma with low grade dysplasia. Anastomotic disruption following ileal pouch anal anastomosis (IPAA) was the major post operative complication. On the twelfth post operative day, laparotomy with thorough peritoneal toileting with repair of disrupted portion with de functioning ileostomy was done.

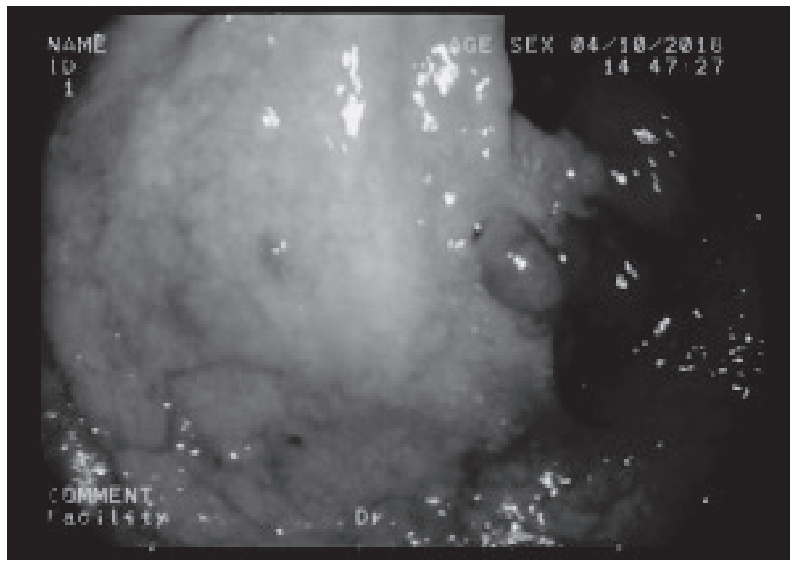

Fig.-1: Showing one large and few tiny polyps in sigmoid region.

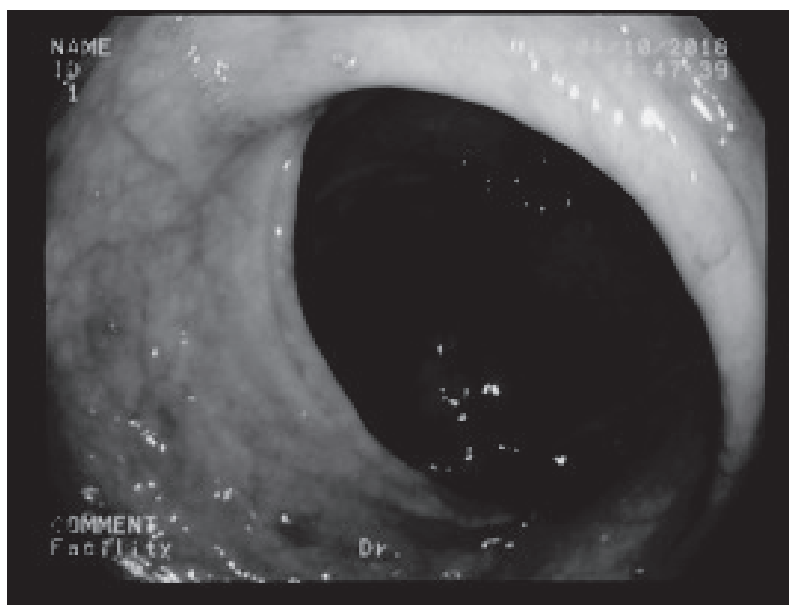

Fig.-2: Shows small sessile polyps and one fungiform polyp in rectum.

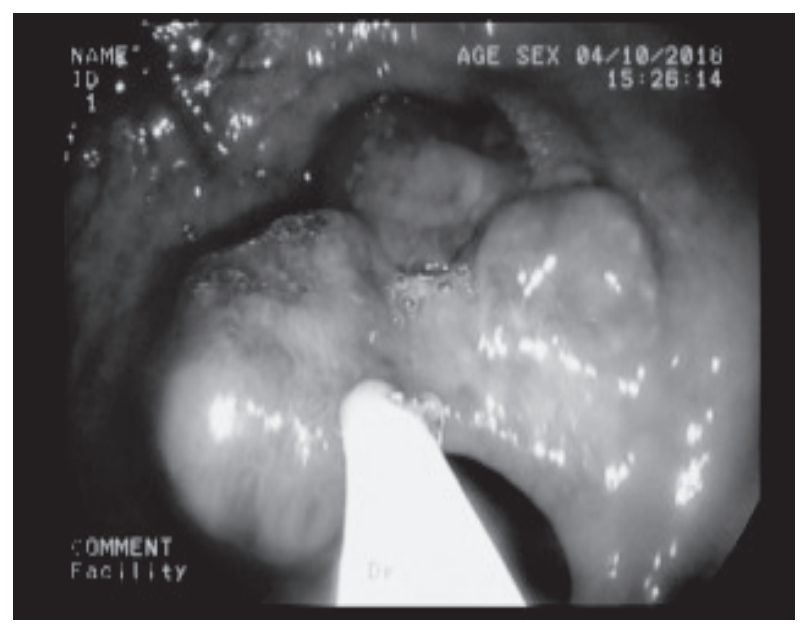

Fig.-3: Showing one large ulcerated polyp occupying the lumen and polypectomy snare. 


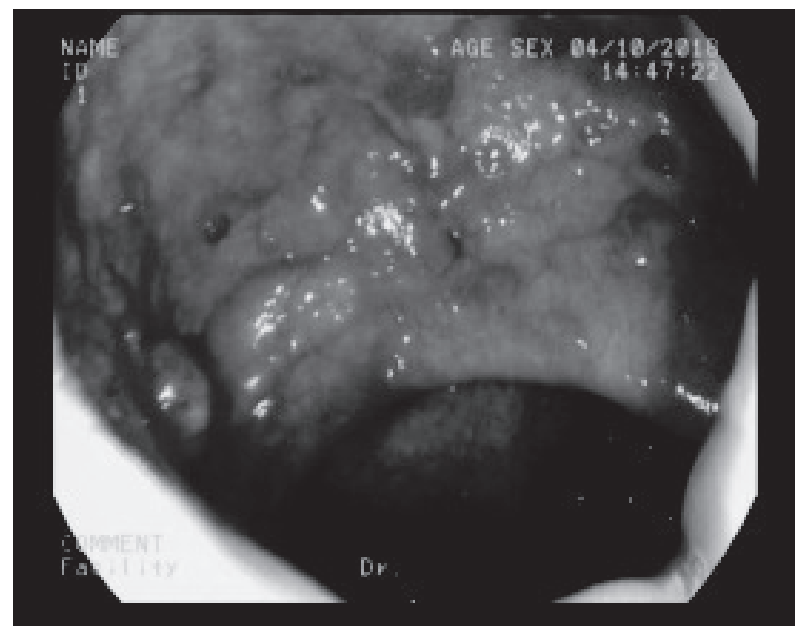

Fig.-4: Polyps of different sizes were seen in rectosigmoid junction.

\section{Discussion:}

Classic FAP is characterized by hundreds to thousands of adenomatous polyposis the colon which usually appearing on average at 16 years of age. ${ }^{7}$ Our patient presented at much earlier age. She had per rectal bleeding since her six years of age. Her mother had similar type of illness. So it indicates autosomal dominant inheritance. Though spontaneous mutation occurs in one third cases. ${ }^{8}$ The abnormal gene can be inherited from either parent or can be the result of a new mutation in the affected individual. The risk of passing the abnormal gene from affected parent to offspring is $50 \%$ for each pregnancy in dominant cases. The risk is the equal for males and females. A retrospective study of outcomes in 492 patients with polyposis found that the age at polyposis onset and years of survival differed significantly by genotype, although the age of onset of colorectal cancer did not. ${ }^{9}$ Colonoscopic examination was done on the patient two years back. But at that time, histopathologic examination revealed juvenile adenomatous polyp without dysplastic changes. As the girl was young and histopathology report showed no dysplastic changes, prophylactic colectomy was postponed at that time. Without colectomy, affected individuals usually develop colorectal cancer by the third or fourth decade of life. In the majority of patients polyps begin to develop during childhood, mostly in the distal colon (rectosigmoid) as small intramucosal nodules and have fewer polyps. In our case, polyps were limited to left colon (descending colon, recto sigmoid region) instead of whole colon. By the time of adolescence, the polyps are usually identified throughout the colon and, thereafter, increase in size and numbers. About half of FAP patients develop adenomas by 15 years of age and $95 \%$ by age 35 years. ${ }^{7}$ Diagnosis of FAP is made in younger people by the presence of the adenomatous polyps and family history of FAP or by genetic testing. ${ }^{5}$ Clinical diagnosis of FAP should be confirmed by genetic testing if possible. However, genetic testing is not possible in our setting. Diagnosis of FAP was made by clinical, histologic features and positive family history in our patient.

FAP is also associated with an increased risk for malignancy of other areas such as the small intestine, thyroid, pancreas, liver, central nervous system (CNS) and bile ducts, but these usually occur in less than $10 \%$ of affected individuals. ${ }^{6}$ Endoscopy of upper GIT was done on this patient to exclude duodenal and gastric polyps. These polyps should be removed timely, if present. Other extra colonic manifestations may present in FAP, like osteomas, dental abnormalities congenital hypertrophy of the retinal pigment epithelium (CHRPE), and soft tissue tumors including epidermoid cysts, fibromas and desmoid tumors. About $5 \%$ of individuals with FAP experience morbidity and/or mortality from desmoid tumors. Individuals with CNS tumors and colorectal polyposis have been defined as Turcot syndrome. The term Gardner syndrome is often used when colonic polyposis is accompanied by clinically obvious osteomas and soft tissue tumors. ${ }^{7}$ Our patient was investigated for extra colonic features and she had no extracolonic features at that time. Attenuated FAP is another unique variant of familial adenomatous polyposis. The disorder is characterized by fewer polyps and later age of onset of polyps, but malignant potential is less than classical FAP. ${ }^{10}$ Extra-colonic manifestations may present in attenuated FAP.

Partial or complete removal of the colon (colectomy) is usually recommended for individuals with classical FAP at an appropriate age, usually between the late teens and late 30s. As histology report of our patient showed dysplastic changes, colectomy was done in her early teens. Development of colon cancer in classic FAP is inevitable if the colon is not removed. An appropriately timed colectomy remains the cornerstone of colon cancer prevention in FAP. ${ }^{11}$ Indications for early surgery include polyps $>10 \mathrm{~mm}$ diameter, polyps with high-grade dysplasia, marked increases in polyp number from one exam to the next, and symptoms. In our case, the size of some polyps was more than $10 \mathrm{~mm}$. Some were ugly looking, fungiform and ulcerated. Surgical options are colectomy with IRA(ileorectal anastomosis) (for $<20$ rectal and $<1,000$ colonic adenomas) and proctocolectomy with IPAA (for severe or profuse adenomas, $>20$ rectal adenomas, and $>1,000$ colonic adenomas). A laparoscopic approach can be used 
for both surgical approaches. Proctocolectomy with ileal pouch anal anastomosis was performed in that girl. Proctocolectomy with ileostomy is rarely needed. $A P C$ mutation location, allowing prediction of severity of rectal polyposis and likelihood of future completion proctectomy, has been suggested as a factor to consider in determining which procedure should be done. ${ }^{12}$ Postsurgical surveillance should include yearly endoscopy of rectum or ileal pouch, and examination of an ileostomy every 2 years. ${ }^{10}$

Colectomy with IRA is a single-stage procedure with slightly less morbidity than the IPAA surgery, but some rectal cancer risk remains and yearly proctoscopy is essential. ${ }^{12,13}$ Even after total proctocolectomy and IPAA, adenomas and cancers may occur in the anal transition zone and in the pouch itself; lifelong endoscopic surveillance is required. ${ }^{14,15}$ Possible morbidities from either surgery include increased bowel frequency and incontinence. Pouch surgery is associated with some loss of fertility in women and some loss of sexual function in men.

\section{Conclusion:}

FAP is one of the most common hereditary syndromes associated with an increased risk of colorectal cancer. Colonic adenocarcinoma is the inevitable consequence of FAP unless the colon is removed. So any child with per rectal bleeding should be asked for family history. Upper gastrointestinal cancers and desmoid tumors are the most common causes of death in patients who have undergone colectomy. So further follow up is necessary after colectomy, as it only limits the risk of colon cancer development.

\section{References:}

1. Perchiniak EM, Groden J. Mechanisms regulating microtubule binding, DNA replication, and apoptosis are controlled by the intestinal tumor suppressor APC. Curr Colorectal Cancer Rep 2011;7:145-51

2. Soravia C, Berk T, Madlensky L,Mitri A, Cheng $\mathrm{H}$, Gallinger S. Genotype-phenotype correlations in attenuated adenomatous polyposis coli. Am J Hum Genet 1998; 62: 1290-1301.

3. Amos-Landgraf J, Kwong LN, Dove WF. A targetselected Apc-mutant rat kindred enhances the modeling of familial human colon cancer. Proc Natl Acad Sci 2007;104: 4036-41.

4. Scheuner MT, McNeel TS, Freedman AN. Population prevalence of familial cancer and common hereditary cancer syndromes. The 2005 California Health Interview Survey. Genet Med 2010;12:726-35.
5. Stoffel EM, Kastrinos F. Familial colorectal cancer, beyond Lynch syndrome. Clin Gastroenterol Hepatol 2014;12:1059-68.

6. Syngal S, Brand RE, Church JM, Giardiello FM, Hampel HL, Burt RW. ACG clinical guideline: Genetic testing and management of hereditary gastrointestinal cancer syndromes. Am J Gastroenterol 2015; 110:223-62.

7. Petersen GM, Slack J, Nakamura Y. Screening guidelines and premorbid diagnosis of familial adenomatous polyposis using linkage. Gastroenterology1991; 100:1658-64.

8. Aretz $S$, Uhlhaas $S$, Caspari R,Mangold E, Pagenstecher C, Propping $P$,et al. Frequency and parental origin of de novo APC mutations in familial adenomatous polyposis. Eur J Hum Genet 2004;12:52-8.

9. Will OC, Hansmann A, Philips RK, Palazzo FF, Meeran K, Marshall M, et al. Adrenal incidentaloma in familial adenomatous polyposis; A long term follow up study and schema for management. Dis Colon Rectum 2009; 52:1637-44.

10. Burt RW, Leppert MF, Slattery ML, Samowitz WS, Spirio LN, Kerber RA, et al. Genetic testing and phenotype in a large kindred with attenuated familial adenomatous polyposis. Gastroenterology 2004; 127:444-51.

11. da Luz Moreira A, Church JM, Burke CA. The evolution of prophylactic colorectal surgery for familial adenomatous polyposis. Dis Colon Rectum 2009; 52:1481-6.

12. Nieuwenhuis $M H$, Bulow $S$, Bjork $J$, Järvinen $H J$, Bülow C, Bisgaard ML,et al. Genotype predicting phenotype in familial adenomatous polyposis: a practical application to the choice of surgery. Dis Colon Rectum 2009;52:1259-63.

13. Kartheuser A, Stangherlin P, Brandt D, Remue C, Sempoux C. Restorative proctocolectomy and ileal pouch-anal anastomosis for familial adenomatous polyposis revisited. Fam Cancer 2006; 5:241-60.

14. Saurin JC, Napoleon B, Gay G,Ponchon T, Arpurt JP, Boustiere C. Endoscopic management of patients with familial adenomatous polyposis (FAP) following a colectomy. Endos-copy 2005; 37:499-501.

15. Smith JC, Schaffer MW, Ballard BR. Adenocarcinomas after prophylactic surgery for familial adenomatous polyposis. J Cancer Ther 2013;4:260-70. 